Supporting Information for

\title{
Facile Solid-State Mechanochemical Synthesis of Eco-Friendly Thermoplastic Polyurethanes and Copolymers Using a Biomass- Derived Furan Diol
}

Changsuk Oh, ${ }^{1}$ Eun Ho Choi, ${ }^{1}$ Eun Jung Choi, ${ }^{1,2}$ Thathan Premkumar, ${ }^{* 1,3}$ and Changsik Song $*, 1$

${ }^{1}$ Department of Chemistry, ${ }^{2}$ Institute of Basic Science and ${ }^{3}$ The University College, Sungkyunkwan University, Suwon, Gyeonggi 16419, Republic of Korea.

*Corresponding authors e-mails: TP: tprem@skku.edu; CS: songcs@skku.edu

\section{Contents:}

$\checkmark$ Table S1. Effect of diisocyanates and catalysts in the mechanochemical PU synthesis (ball mill) with BHMF

$\checkmark$ Fig. S1. ${ }^{1}$ H-NMR spectra of BHMF (monomer) and FR-PU $-\mathrm{S} 3$

$\checkmark \quad$ Fig S2. FTIR spectra of FR-co-PUs S4

$\checkmark \quad$ Fig S3. ${ }^{1} \mathrm{H}$ NMR spectrum of FR-co-PU1b -S5

$\checkmark$ Table S2. Polymerization results of copolymer FR-co-PUs according to ball milling reaction time S6

Fig S4. Temperature dependences of the storage modulus, loss modulus, and phase angle of FR-PU and FR-co-PU1b S7 
Table S1. Effect of diisocyanates and catalysts in the mechanochemical PU synthesis (ball mill) with BHMF

\begin{tabular}{c|cccccccc}
\hline Entry & Diisocyanate & Catalyst & $\begin{array}{c}\text { Frequency } \\
(\mathbf{H z})\end{array}$ & $\begin{array}{c}\text { Time } \\
(\mathbf{m i n})\end{array}$ & $\begin{array}{c}\text { Conversion } \\
(\mathbf{\%})\end{array}$ & $\mathbf{M}_{\mathbf{n}}$ & $\mathbf{M}_{\mathbf{w}}$ & $\begin{array}{c}\text { PDI } \\
\left(\mathbf{M}_{\mathbf{w} /} \mathbf{M}_{\mathbf{n}}\right.\end{array}$ \\
\hline 1 & HDI & DBTDL & 20 & 60 & 98 & $9 \mathrm{k}$ & $12 \mathrm{k}$ & 1.40 \\
2 & TDI & DBTDL & 20 & 60 & 99 & $14 \mathrm{k}$ & $29 \mathrm{k}$ & 2.01 \\
3 & MDI & DBTDL & 20 & 60 & 97 & $59 \mathrm{k}$ & $163 \mathrm{k}$ & 2.75 \\
4 & MDI & DABCO & 20 & 60 & 98 & $56 \mathrm{k}$ & $83 \mathrm{k}$ & 1.50 \\
5 & MDI & DBU & 20 & 60 & 99 & $40 \mathrm{k}$ & $57 \mathrm{k}$ & 1.40 \\
6 & MDI & $\mathrm{X}$ & 20 & 60 & 61 & $4 \mathrm{k}$ & $5 \mathrm{k}$ & 1.18 \\
\hline
\end{tabular}




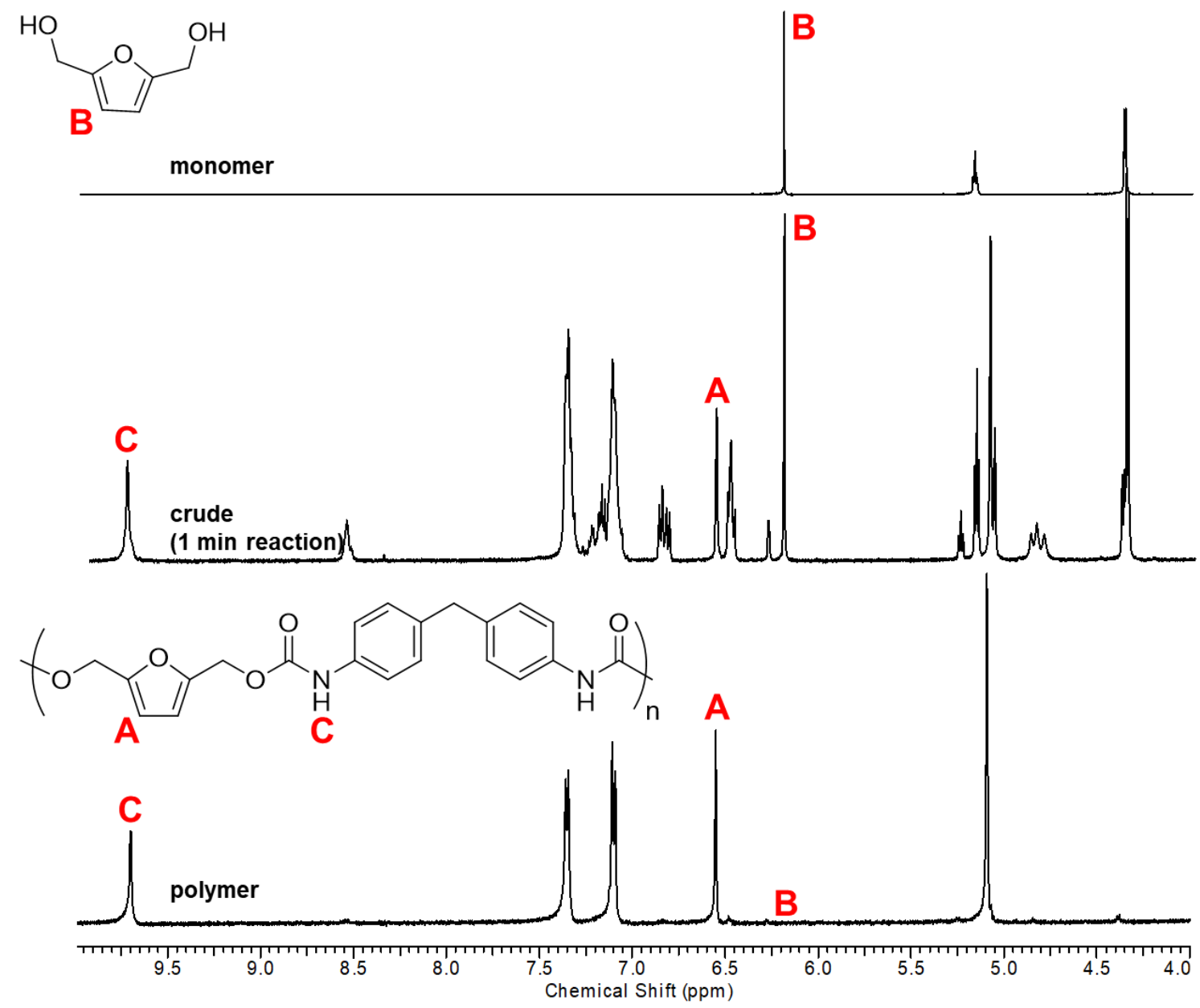

Figure S1. ${ }^{1} \mathrm{H}-\mathrm{NMR}$ spectra of BHMF (monomer), the crude mixture of ball-milling for 1 min, and isolated FR-PU. The reaction conversion was calculated by comparing the ratio between integration for proton $\mathbf{B}$ from the monomer and those for proton $\mathbf{A}$ from the polymer. 

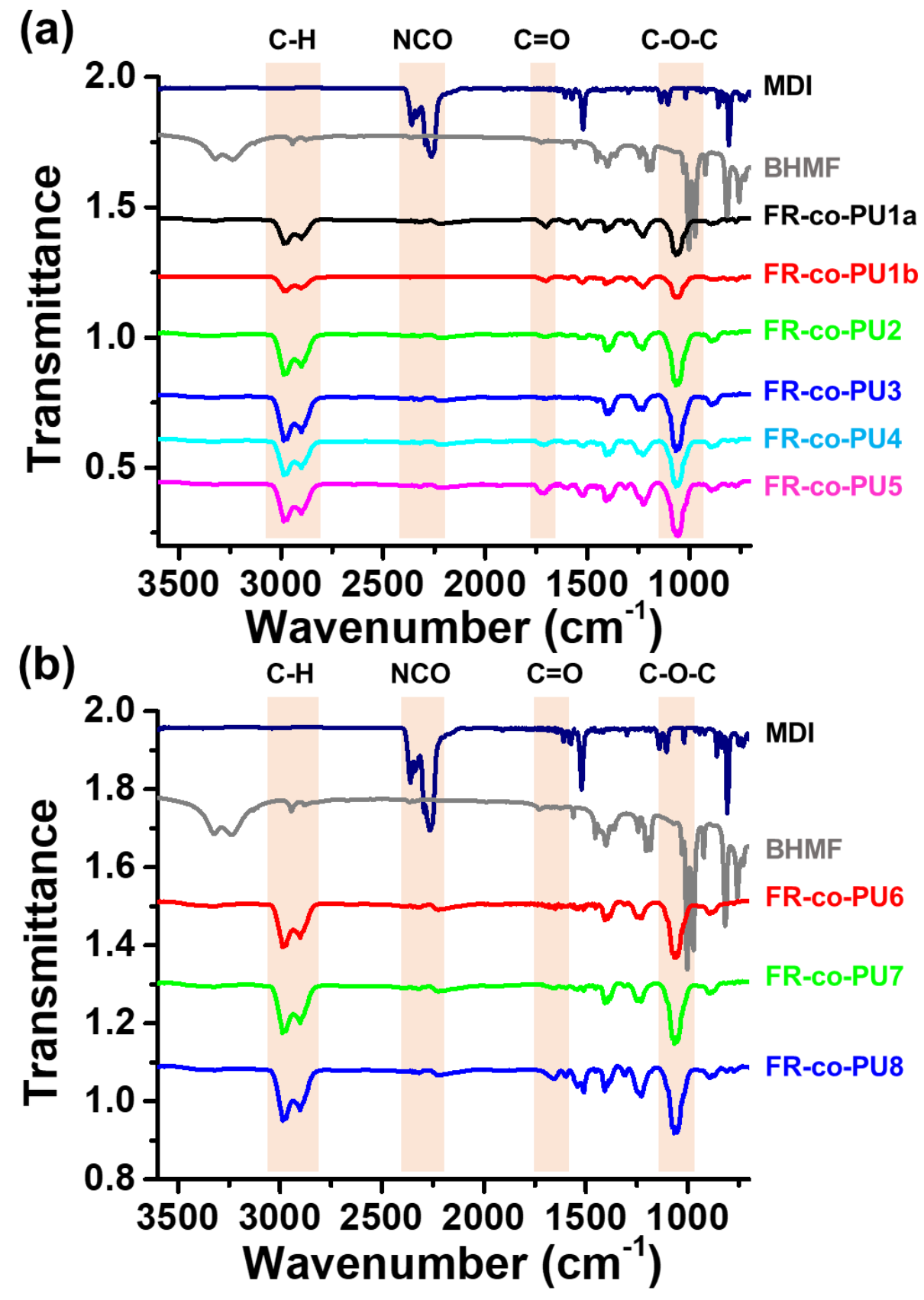

Figure S2. FTIR spectra of (a) PU copolymer using diol and (b) PU copolymer using diamine 


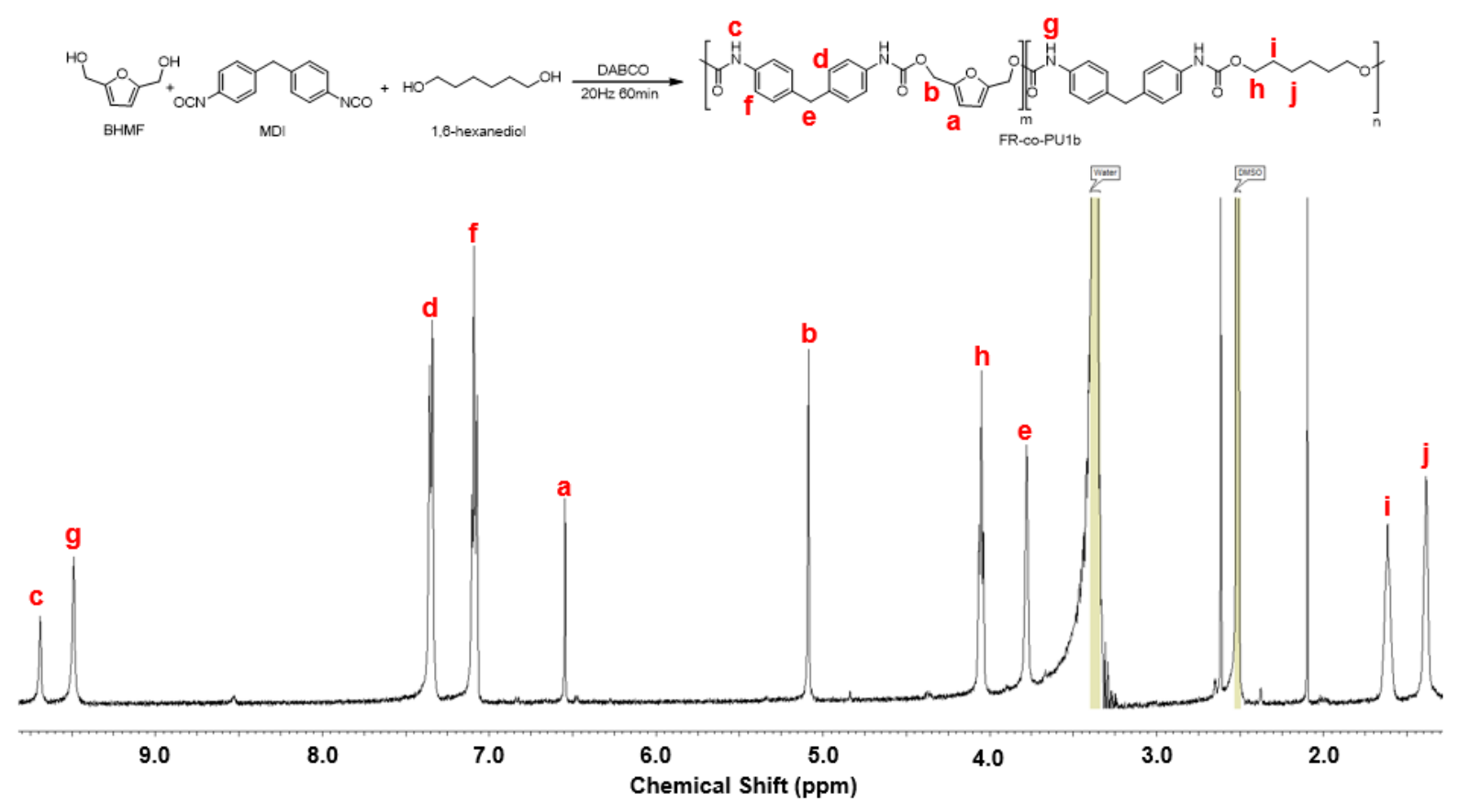

Figure S3. ${ }^{1} \mathrm{H}$ NMR spectrum of FR-co-PU1b 
Table S2. Polymerization results of copolymer FR-co-PUs according to ball milling reaction time

\begin{tabular}{|c|c|c|c|c|c|c|c|}
\hline Entry & Diol/ diamine & $\begin{array}{c}\text { Frequency } \\
(\mathbf{H z})\end{array}$ & $\begin{array}{l}\text { Time } \\
\text { (min) }\end{array}$ & $\begin{array}{c}\text { Conversion } \\
(\%)\end{array}$ & $\mathbf{M}_{\mathbf{n}}$ & $\mathbf{M}_{\mathbf{w}}$ & $\begin{array}{c}\text { PDI } \\
\left(\mathbf{M}_{\mathbf{w} /} \mathbf{M}_{\mathbf{n})}\right.\end{array}$ \\
\hline 1 & 1,6-Hexanediol & 20 & 60 & 94 & $45 \mathrm{k}$ & $75 \mathrm{k}$ & 1.67 \\
\hline 2 & 1,6-Hexanediol & 20 & 90 & 96 & $12 \mathrm{k}$ & $19 \mathrm{k}$ & 1.60 \\
\hline 3 & 1,4-Butanediol & 20 & 60 & 97 & $28 \mathrm{k}$ & $49 \mathrm{k}$ & 1.74 \\
\hline 4 & 1,4-Butanediol & 20 & 90 & 96 & $13 k$ & $18 \mathrm{k}$ & 1.42 \\
\hline 5 & 1,3-Propanediol & 20 & 60 & 95 & $15 \mathrm{k}$ & $22 \mathrm{k}$ & 1.52 \\
\hline 6 & 1,3-Propanediol & 20 & 90 & 96 & $14 \mathrm{k}$ & $20 \mathrm{k}$ & 1.46 \\
\hline 7 & Ethylene glycol & 20 & 60 & 98 & $69 \mathrm{k}$ & $111 \mathrm{k}$ & 1.62 \\
\hline 8 & Ethylene glycol & 20 & 90 & 97 & $59 \mathrm{k}$ & $102 \mathrm{k}$ & 1.72 \\
\hline 9 & Isosorbide & 20 & 60 & 97 & $23 \mathrm{k}$ & $35 \mathrm{k}$ & 1.50 \\
\hline 10 & Isosorbide & 20 & 90 & 98 & $26 \mathrm{k}$ & $40 \mathrm{k}$ & 1.57 \\
\hline 11 & 1,6-Diaminohexane & 20 & 60 & 34 & $11 \mathrm{k}$ & $14 \mathrm{k}$ & 1.30 \\
\hline 12 & 1,6-Diaminohexane & 20 & 90 & 25 & $10 \mathrm{k}$ & $13 \mathrm{k}$ & 1.25 \\
\hline 13 & 1,4-Diaminobutane & 20 & 60 & 39 & $14 \mathrm{k}$ & $21 \mathrm{k}$ & 1.44 \\
\hline 14 & 1,4-Diaminobutane & 20 & 90 & 37 & $13 \mathrm{k}$ & $19 \mathrm{k}$ & 1.40 \\
\hline 15 & Ethylenediamine & 20 & 60 & 76 & $20 \mathrm{k}$ & $34 \mathrm{k}$ & 1.75 \\
\hline 16 & Ethylenediamine & 20 & 90 & 71 & $18 \mathrm{k}$ & $31 \mathrm{k}$ & 1.70 \\
\hline
\end{tabular}


A

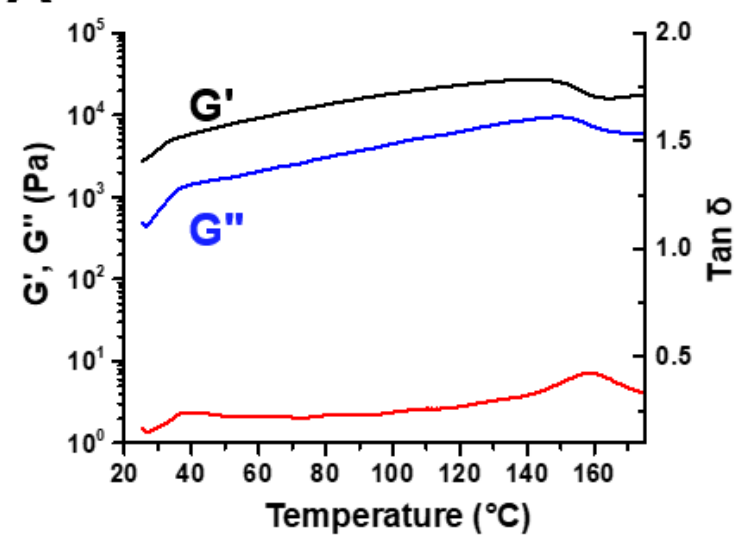

B

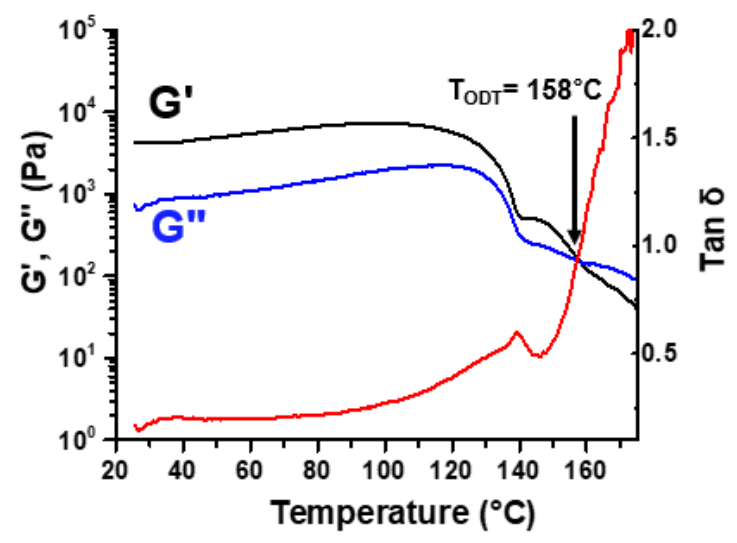

Figure S4. Temperature dependences of the storage modulus, G', loss modulus, G", and phase angle, $\delta$, of (A) FR-PU and (B) FR-co-PU1b. 